\title{
Existence of Solutions for Boundary Value Problems of Vibration Equation with Fractional Derivative
}

\author{
Nan Yao, Zeyu Luo \\ College of Science, University of Shanghai for Science and Technology, Shanghai, China \\ Email: 1491771426@qq.com, 403141236@qq.com
}

How to cite this paper: Yao, N. and Luo, Z.Y. (2019) Existence of Solutions for Boundary Value Problems of Vibration Equation with Fractional Derivative. Journal of Applied Mathematics and Physics, 7 , 1067-1076.

https://doi.org/10.4236/jamp.2019.75072

Received: April 8, 2019

Accepted: May 20, 2019

Published: May 23, 2019

Copyright $\odot 2019$ by author(s) and Scientific Research Publishing Inc. This work is licensed under the Creative Commons Attribution International License (CC BY 4.0).

http://creativecommons.org/licenses/by/4.0/

\begin{abstract}
In this paper, we investigate the solvability of boundary value problems for a class of vibration differential equation describing the fractional order damped system with signal stimulus. By presenting kernel function through the Laplace transform, and using the eigenvalue and the improved Leray-Schauder degree, the existence of solutions for boundary value problems is established.
\end{abstract}

\section{Keywords}

Caputo Derivative, Boundary Value Problems for Vibration Equation, Laplace Transform, Eigenvalue, Improved Leray-Schauder Degree

\section{Introduction}

Fractional calculus has been applied more and more widely in many fields of science and engineering, many scholars have done a lot of research on it [1]-[8]. When describing fractional Brownian motion in Anomalous diffusion, if time fractional differential operator is introduced, fractional Langevin equation [9]

$$
x^{\prime \prime}(t)+\gamma \frac{\mathrm{d}^{\alpha-1}}{\mathrm{~d} t^{\alpha-1}} x^{\prime}(t)=\Gamma(t)
$$

is obtained. The fractional Langevin equation describes both subdiffusion for $0<\alpha<1$ and superdiffusion for $1<\alpha<2$.

Fractional differential equations are also used to describe damped vibrations in viscoelastic media. In [10], Podlubny studied the initial value problem for the inhomogeneous Bagley-Torvik equation 


$$
\left\{\begin{array}{l}
A y^{\prime \prime}(t)+B_{0} D_{t}^{\frac{3}{2}} y(t)+C y(t)=f(t), t>0, \\
y(0)=0, y^{\prime}(0)=0
\end{array}\right.
$$

and the numerical solutions are presented. The numerical solution is in agreement with the analytical solution, obtained with the help of the fractional Green's function for a three-term fractional differential equation with constant coefficients.

Motivated by the above works, we study the following boundary value problems for a class of vibration differential equation describing the fractional order damped system with signal stimulus

$$
\left\{\begin{array}{l}
x^{\prime \prime}(t)-b^{c} D_{0^{+}}^{\alpha} x(t)=f(t, x(t)), t \in(0,1), \\
x(0)=0, x^{\prime}(1)=c x^{\prime}(0),
\end{array}\right.
$$

where $0<b<1,1<\alpha<2, c \geq 0,{ }^{c} D_{0^{+}}^{\alpha}$ is the Caputo fractional derivative of order $\alpha, f:[0,1] \times \mathbb{R} \rightarrow(0, \infty)$ is continuous. Moreover, $x^{\prime \prime}(t)$ indicates the ratio of inertia force to mass, ${ }^{c} D_{0^{+}}^{\alpha} x(t)$ is external damping term, namely, dissipative term, $f$ represents the external force.

By using the Laplace transform, the kernel function is obtained. And then, by using the eigenvalue and the improved Leray-Schauder degree, the existence of the solutions to boundary value problem (1.1) is proved, see Theorem 1. So we can investigate the state of the oscillator motion under this system.

\section{Preliminaries}

In this part, we recall some definitions and lemmas which are critical to the existence result. The definitions of fractional integral and fractional derivative can be found in [11] [12].

Definition 1. [10] Assume that function $g(t)$ is defined in $(0, \infty)$, then the Laplace transform of $g(t)$ is defined as

$$
\tilde{g}(p)=L[g(t)](p)=\int_{0}^{\infty} \mathrm{e}^{-p t} g(t) \mathrm{d} t, p>0,
$$

as long as the generalized integral is convergent.

Definition 2. [10] The original $g(t)$ can be restored from the Laplace transform $\tilde{g}(p)$ with the help of the inverse Laplace transform

$$
g(t)=L^{-1}[\tilde{g}(p)]=\frac{1}{2 \pi i} \int_{\sigma-i \infty}^{\sigma+i \infty} \tilde{g}(p) \mathrm{e}^{p t} \mathrm{~d} p, \sigma=\operatorname{Re}(p)>\sigma_{0} .
$$

where $\sigma_{0}$ lies in the right half plane of the absolute convergence of the Laplace integral.

Definition 3. [12] Let $\delta, \beta>0$. The function

$$
E_{\delta, \beta}(z)=\sum_{k=0}^{\infty} \frac{z^{k}}{\Gamma(\delta k+\beta)},
$$

whenever the series converges is called the two-parameter Mittag-Leffler function with parameters $\delta$ and $\beta$. 
Lemma 1. [11] Let $l-1<\delta \leq l, l \in \mathbb{N}$. The Laplace transform formula for ${ }^{c} D_{0^{+}}^{\delta} g(t)$ is

$$
L\left[{ }^{c} D_{0^{+}}^{\delta} g(t)\right](p)=p^{\delta} L[g(t)](p)-\sum_{j=0}^{l-1} p^{\delta-1-j} g^{(j)}(0), p>0 .
$$

Let

$$
E_{\delta, \beta}^{(k)}(z)=\frac{\mathrm{d}^{k}}{\mathrm{~d} z^{k}} E_{\delta, \beta}(z), k=0,1,2, \cdots .
$$

Lemma 2. [12] Let $\delta, \beta>0$. The power series $E_{\delta, \beta}(z)$ is convergent for all $z \in \mathbb{C}$. In other words, $E_{\delta, \beta}(z)$ is an entire function.

Lemma 3. Let $z \in \mathbb{R}, k=0,1,2, \cdots$. Then

$$
E_{\delta, \beta}^{(k)}(z)=\sum_{j=0}^{\infty} \frac{z^{j} \Gamma(k+j+1)}{\Gamma(j+1) \Gamma(\delta(k+j)+\beta)} .
$$

Proof. By Definition 3 and Lemma 2, we can get

$$
\begin{aligned}
E_{\delta, \beta}^{(k)}(z) & =\sum_{j=0}^{\infty} \frac{\mathrm{d}^{k}}{\mathrm{~d} z^{k}}\left(\frac{z^{j}}{\Gamma(\delta j+\beta)}\right) \\
& =\sum_{j=k}^{\infty} \frac{\mathrm{d}^{k}}{\mathrm{~d} z^{k}}\left(\frac{\Gamma(j+1) z^{j-k}}{\Gamma(j-k+1) \Gamma(\delta j+\beta)}\right) \\
& =\sum_{j=0}^{\infty} \frac{z^{j} \Gamma(k+j+1)}{\Gamma(j+1) \Gamma(\delta(k+j)+\beta)} .
\end{aligned}
$$

Thus, the lemma can be obtained.

Lemma 4. [11] Let $\delta, \beta>0, E_{\delta, \beta}(z)$ be a two-parameter Mittag-Leffler function. Then

$$
L\left[t^{\delta k+\beta-1} E_{\delta, \beta}^{(k)}\left( \pm a t^{\delta}\right)\right](p)=\frac{k ! p^{\delta-\beta}}{\left(p^{\delta} \mp a\right)^{k+1}}, p>|a|^{\frac{1}{\delta}} .
$$

Denote

$$
\begin{aligned}
& g_{1}(t)=t E_{2-\alpha, 2}\left(b t^{2-\alpha}\right), \\
& g_{2}(t)=\frac{1}{b}\left(E_{2-\alpha, 1}\left(b t^{2-\alpha}\right)-1\right), t \in[0, \infty) .
\end{aligned}
$$

Lemma 5. The functions $g_{1}$ and $g_{2}$, defined above, have the following properties.

1) $g_{1}(t)$ and $g_{2}(t)$ are represented by absolutely and uniformly convergent series and $g_{1}(t), g_{2}(t) \geq 0$ on $[0,1]$;

2) $g_{1}^{\prime}(t)=E_{2-\alpha, 1}\left(b t^{2-\alpha}\right)>1$;

3) $g_{1}^{\prime}(t)-b g_{2}(t)=1$.

Proof. 1) By Lemma 2, we can show $g_{1}(t)$ is an entire function. Thus, $g_{1}(t)$ is represented by absolutely and uniformly convergent series on $[0,1]$.

Similarly, $g_{2}(t)$ is also represented by absolutely and uniformly convergent series on $[0,1]$. And we can easily have $g_{1}(t), g_{2}(t) \geq 0$.

2) In view of $g_{1}(t)$ is represented by absolutely and uniformly convergent 
series on $[0,1]$,

$$
g_{1}^{\prime}(t)=\sum_{n=0}^{\infty} \frac{b^{n} t^{(2-\alpha) n}}{\Gamma((2-\alpha) n+1)}=E_{2-\alpha, 1}\left(b t^{2-\alpha}\right)>1 .
$$

3) From the definition of $g_{1}^{\prime}(t)$, we have

$$
g_{1}^{\prime}(t)-b g_{2}(t)=E_{2-\alpha, 1}\left(b t^{2-\alpha}\right)-b \cdot \frac{1}{b}\left(E_{2-\alpha, 1}\left(b t^{2-\alpha}\right)-1\right)=1 .
$$

The proof is complete.

Lemma 6. For $y(t)$ is continuous on $[0,1]$ and $c-g_{1}^{\prime}(1) \neq 0$, the unique solution of

$$
\left\{\begin{array}{l}
x^{\prime \prime}(t)-b^{c} D_{0^{+}}^{\alpha} x(t)=y(t), t \in(0,1), \\
x(0)=0, x^{\prime}(1)=c x^{\prime}(0)
\end{array}\right.
$$

is

$$
x(t)=\int_{0}^{1} G(t, s) y(s) \mathrm{d} s, t \in[0,1]
$$

where

$$
G(t, s)= \begin{cases}\frac{g_{1}(t) g_{1}^{\prime}(1-s)}{c-g_{1}^{\prime}(1)}+g_{1}(t-s), & 0 \leq s \leq t \leq 1, \\ \frac{g_{1}(t) g_{1}^{\prime}(1-s)}{c-g_{1}^{\prime}(1)}, & 0 \leq t<s \leq 1 .\end{cases}
$$

Proof. By Lemma 1, we have

$$
\begin{aligned}
& L\left[x^{\prime \prime}(t)\right](p)=p^{2} L[x(t)](p)-x(0) p-x^{\prime}(0), \\
& L\left[{ }^{c} D_{0^{+}}^{\alpha} x(t)\right](p)=p^{\alpha} L[x(t)](p)-x(0) p^{\alpha-1} .
\end{aligned}
$$

Apply Laplace transform to both sides of $x^{\prime \prime}(t)-b^{c} D_{0^{+}}^{\alpha} x(t)=y(t)$, we can easily obtain

$$
p^{2} L[x(t)](p)-x(0) p-x^{\prime}(0)-b\left(p^{\alpha} L[x(t)](p)-x(0) p^{\alpha-1}\right)=L[y(t)](p),
$$

namely,

$$
L[x(t)](p)=\frac{x(0) p+x^{\prime}(0)-b x(0) p^{\alpha-1}+L[y(t)](p)}{p^{2}-b p^{\alpha}} .
$$

If $\left|b p^{\alpha-2}\right|<1$, we have

$$
\frac{1}{p^{2}-b p^{\alpha}}=\frac{p^{-2}}{1-b p^{\alpha-2}}=p^{-2} \sum_{n=0}^{\infty}\left(b p^{\alpha-2}\right)^{n}=\sum_{n=0}^{\infty} b^{n} p^{n(\alpha-2)-2},
$$

By virtue of Lemma 4, we can show

$$
\frac{1}{p^{2}-b p^{\alpha}}=\sum_{n=0}^{\infty} b^{n} p^{n(\alpha-2)-2}=L\left[g_{1}(t)\right](p), p>0 .
$$

Similarly, if $b p^{\alpha-2}<1$ and $p>0$, we have

$$
\begin{aligned}
& \frac{p}{p^{2}-b p^{\alpha}}=\sum_{n=0}^{\infty} b^{n} p^{n(\alpha-2)-1}=L\left[g_{1}^{\prime}(t)\right](p), \\
& \frac{p^{\alpha-1}}{p^{2}-b p^{\alpha}}=\sum_{n=0}^{\infty} b^{n} p^{n(\alpha-2)+\alpha-3}=L\left[g_{2}(t)\right](p) .
\end{aligned}
$$


So (2.4) is equivalent to

$$
\begin{aligned}
L[x(t)](p)= & x(0) L\left[g_{1}^{\prime}(t)\right](p)+x^{\prime}(0) L\left[g_{1}(t)\right](p) \\
& -b x(0) L\left[g_{2}(t)\right](p)+L[y(t)](p) L\left[g_{1}(t)\right](p) .
\end{aligned}
$$

Furthermore,

$$
L[y(t)](p) L\left[g_{1}(t)\right](p)=L\left[\int_{0}^{t} g_{1}(t-s) y(s) \mathrm{d} s\right](p),
$$

then we can get the inverse Laplace transform for $(2.5)$ is

$$
\begin{gathered}
x(t)=x(0) g_{1}^{\prime}(t)+x^{\prime}(0) g_{1}(t)-b x(0) g_{2}(t)+\int_{0}^{t} g_{1}(t-s) y(s) \mathrm{d} s \\
=x(0)\left(g_{1}^{\prime}(t)-b g_{2}(t)\right)+x^{\prime}(0) g_{1}(t)+\int_{0}^{t} g_{1}(t-s) y(s) \mathrm{d} s \\
=x(0)+x^{\prime}(0) g_{1}(t)+\int_{0}^{t} g_{1}(t-s) y(s) \mathrm{d} s
\end{gathered}
$$

Substituting (2.7) into (2.6), we get

$$
x(t)=\int_{0}^{1} G(t, s) y(s) \mathrm{d} s,
$$

where $G(t, s)$ is defined by (2.3).

On the other hand, by using the above proof, if $x=x(t)$ satisfies (2.2), we obtain that $x$ satisfies $x^{\prime \prime}(t)-b^{c} D_{0^{+}}^{\alpha} x(t)=y(t)$ and $x(0)=0, x^{\prime}(1)=c x^{\prime}(0)$. The proof is complete.

Lemma 7. The eigenfunction of

$$
\left\{\begin{array}{l}
x^{\prime \prime}(t)-b^{c} D_{0^{+}}^{\alpha} x(t)=\lambda x(t), t \in(0,1), \\
x(0)=0, x^{\prime}(1)=c x^{\prime}(0)
\end{array}\right.
$$

is $x(t)=K \sum_{n=0}^{\infty} \frac{\lambda^{n}}{n !} t^{2 n+1} E_{2-\alpha, 2+\alpha n}^{(n)}\left(b t^{2-\alpha}\right)$ and its corresponding eigenvalue $\lambda$ is the solution of equation

$$
c=\sum_{n=0}^{\infty} \frac{\lambda^{n}}{n !} E_{2-\alpha, 1+\alpha n}^{(n)}(b),
$$

where $K$ is a constant and $\lambda>0$.

Proof. Let $x=x(t)$ is the solution of boundary value problem (2.8). Apply Laplace transform to both sides of $x^{\prime \prime}(t)-b^{c} D_{0^{+}}^{\alpha} x(t)=\lambda x(t)$, we can easily obtain

$$
\begin{gathered}
L[x(t)](p)=\frac{x(0) p+x^{\prime}(0)-b x(0) p^{\alpha-1}}{p^{2}-\lambda-b p^{\alpha}} . \\
\text { If }\left|\frac{\lambda}{p^{2}-b p^{\alpha}}\right|<1 \text {, we have } \\
\frac{1}{p^{2}-\lambda-b p^{\alpha}}=\frac{p^{-\alpha}}{p^{2-\alpha}-b} \cdot \frac{1}{1-\frac{\lambda p^{-\alpha}}{p^{2-\alpha}-b}}=\sum_{n=0}^{\infty} \frac{\lambda^{n} p^{-\alpha(n+1)}}{\left(p^{2-\alpha}-b\right)^{n+1}} .
\end{gathered}
$$


By virtue of Lemma 4, we can show, if $p>\lambda^{\frac{1}{2-\alpha}}$,

$$
\frac{1}{p^{2}-\lambda-b p^{\alpha}}=\sum_{n=0}^{\infty} \frac{\lambda^{n} p^{-\alpha(n+1)}}{\left(p^{2-\alpha}-b\right)^{n+1}}=L\left[\sum_{n=0}^{\infty} \frac{\lambda^{n}}{n !} t^{2 n+1} E_{2-\alpha, 2+\alpha n}^{(n)}\left(b t^{2-\alpha}\right)\right](p) \text {. }
$$

Similarly, if $\left|\frac{\lambda}{p^{2}-b p^{\alpha}}\right|<1$ and $p>\lambda^{\frac{1}{2-\alpha}}$

$$
\begin{aligned}
& \frac{p}{p^{2}-\lambda-b p^{\alpha}}=\sum_{n=0}^{\infty} \frac{\lambda^{n} p^{1-\alpha(n+1)}}{\left(p^{2-\alpha}-b\right)^{n+1}}=L\left[\sum_{n=0}^{\infty} \frac{\left(\lambda t^{2}\right)^{n}}{n !} E_{2-\alpha, 1+\alpha n}^{(n)}\left(b t^{2-\alpha}\right)\right](p), \\
& \frac{p^{\alpha-1}}{p^{2}-\lambda-b p^{\alpha}}=\sum_{n=0}^{\infty} \frac{\lambda^{n} p^{-\alpha n-1}}{\left(p^{2-\alpha}-b\right)^{n+1}}=L\left[\sum_{n=0}^{\infty} \frac{\lambda^{n}}{n !} t^{2 n+2-\alpha} E_{2-\alpha, 3-\alpha+\alpha n}^{(n)}\left(b t^{2-\alpha}\right)\right](p) .
\end{aligned}
$$

So (2.9) is equivalent to

$$
\begin{aligned}
L[x(t)](p)= & x^{\prime}(0) L\left[\sum_{n=0}^{\infty} \frac{\lambda^{n}}{n !} t^{2 n+1} E_{2-\alpha, 2+\alpha n}^{(n)}\left(b t^{2-\alpha}\right)\right](p) \\
& +x(0) L\left[\sum_{n=0}^{\infty} \frac{\left(\lambda t^{2}\right)^{n}}{n !} E_{2-\alpha, 1+\alpha n}^{(n)}\left(b t^{2-\alpha}\right)\right](p) \\
& -b x(0) L\left[\sum_{n=0}^{\infty} \frac{\lambda^{n}}{n !} t^{2 n+2-\alpha} E_{2-\alpha, 3-\alpha+\alpha n}^{(n)}\left(b t^{2-\alpha}\right)\right](p) .
\end{aligned}
$$

Furthermore, we can get the inverse Laplace transform for (2.10) is

$$
\begin{aligned}
x(t)= & x^{\prime}(0) \sum_{n=0}^{\infty} \frac{\lambda^{n}}{n !} t^{2 n+1} E_{2-\alpha, 2+\alpha n}^{(n)}\left(b t^{2-\alpha}\right)+x(0) \sum_{n=0}^{\infty} \frac{\left(\lambda t^{2}\right)^{n}}{n !} E_{2-\alpha, 1+\alpha n}^{(n)}\left(b t^{2-\alpha}\right) \\
& -b x(0) \sum_{n=0}^{\infty} \frac{\lambda^{n}}{n !} t^{2 n+2-\alpha} E_{2-\alpha, 3-\alpha+\alpha n}^{(n)}\left(b t^{2-\alpha}\right) .
\end{aligned}
$$

Because $x(0)=0, x^{\prime}(1)=c x^{\prime}(0)$, we can show

$$
\begin{aligned}
& x(t)=K \sum_{n=0}^{\infty} \frac{\lambda^{n}}{n !} t^{2 n+1} E_{2-\alpha, 2+\alpha n}^{(n)}\left(b t^{2-\alpha}\right), \\
& c=\sum_{n=0}^{\infty} \frac{\lambda^{n}}{n !} E_{2-\alpha, 1+\alpha n}^{(n)}(b) .
\end{aligned}
$$

The proof is complete.

Lemma 8. The function $G(t, s)$ defined by (2.3) is continuous on $[0,1] \times[0,1]$.

Proof. By the definition of $G(t, s)$ and Lemma 5, we get $G(t, s)$ is continuous for $(t, s) \in[0,1] \times[0,1]$. The proof is complete.

\section{The Existence of the Solutions}

Let

$$
\beta=\lim _{x \rightarrow \infty} \sup _{t \in[0,1]} \frac{f(t, x)}{x} .
$$


Throughout this paper, we always suppose that the following conditions are satisfied.

(H1) There exists constant $r>0, \tau \in(0,1)$ such that $f(\cdot, x)>\frac{r}{\tau g_{1}(1-\tau)}$ for any $|x|<r$.

(H2) There exists $\lambda>0$ such that $\lambda \neq \beta$, here $\lambda$ satisfies

$$
c=\sum_{n=0}^{\infty} \frac{\lambda^{n}}{n !} E_{2-\alpha, 1+\alpha n}^{(n)}(b) .
$$

Let $E=C[0,1]$, with the norm $\|x\|=\max _{t \in[0,1]}|x(t)|$. Obviously, $(E,\|\cdot\|)$ is a Banach space.

Define the operators $T, A: E \rightarrow E$,

$$
\begin{aligned}
& T x(t)=\int_{0}^{1} G(t, s) x(s) \mathrm{d} s, \\
& A x(t)=\int_{0}^{1} G(t, s) f(s, x(s)) \mathrm{d} s .
\end{aligned}
$$

By virtue of Lemma 6, the solution of boundary value problem (1.1) is equivalent to the fixed point of the operator $A$; Boundary value problem (2.8) is equivalent to the following integral equation

$$
x(t)=\lambda \int_{0}^{1} G(t, s) x(s) \mathrm{d} s .
$$

Therefore, $\lambda T x=x$, we have $\frac{1}{\lambda}$ is the eigenvalue of operator $T$ corresponding to the eigenfunction (3.1).

Lemma 9. $A: E \rightarrow E$ is completely continuous.

Proof. Let $G_{0}=\max _{t, s \in[0,1]}|G(t, s)|$. Obviously, $A: E \rightarrow E$.

Let $\left\{x_{n}\right\} \subset E, x \in E$ such that $\left\|x_{n}-x\right\| \rightarrow 0$ as $n \rightarrow \infty$. So there exists $\rho>0$ such that $\left\|x_{n}\right\| \leq \rho,\|x\| \leq \rho$.

Let $D=\{x \in E ;\|x\| \leq \rho\}, M=\max _{t \in[0,1], x \in[-\rho, \rho]} f(t, x)$. Then

$$
\begin{aligned}
&\left|A x_{n}(t)-A x(t)\right|=\left|\int_{0}^{1} G(t, s)\left(f\left(s, x_{n}(s)\right)-f(s, x(s))\right) \mathrm{d} s\right| \\
& \leq \int_{0}^{1}\left|G(t, s) f\left(s, x_{n}(s)\right)-f(s, x(s))\right| \mathrm{d} s, \\
&\left|G(t, s) f\left(s, x_{n}(s)\right)-f(s, x(s))\right| \leq 2 M G_{0}
\end{aligned}
$$

By virtue of Lebesgue's dominated convergence theorem, we have

$$
\begin{aligned}
& \lim _{n \rightarrow \infty} \int_{0}^{1}\left|G(t, s) f\left(s, x_{n}(s)\right)-f(s, x(s))\right| \mathrm{d} s \\
& =\int_{0}^{1}|G(t, s)| \lim _{n \rightarrow \infty}\left|f\left(s, x_{n}(s)\right)-f(s, x(s))\right| \mathrm{d} s \\
& =0,
\end{aligned}
$$

so $\left\|A x_{n}-A x\right\| \rightarrow 0$ as $n \rightarrow+\infty$. Hence, the operator $A$ is continuous.

For each $x$ in the bounded area $D$,

$$
|A x(t)|=\left|\int_{0}^{1} G(t, s) f(s, x(s)) \mathrm{d} s\right| \leq G_{0} M .
$$


Consequently, the operator $A$ is uniformly bounded.

By the continuity of $G(t, s)$ on $[0,1] \times[0,1], \forall \varepsilon>0, \exists \delta>0$ for any $t_{1}, t_{2}, s_{1}, s_{2} \in[0,1]$, if $\left|t_{1}-t_{2}\right|<\delta,\left|s_{1}-s_{2}\right|<\delta$, then we have

$$
\left|G\left(t_{1}, s_{1}\right)-G\left(t_{2}, s_{2}\right)\right|<\varepsilon \text {. }
$$

If $\left|t_{1}-t_{2}\right|<\delta, x \in D$, we obtain

$$
\left|A x\left(t_{2}\right)-A x\left(t_{1}\right)\right|=\left|\int_{0}^{1}\left(G\left(t_{2}, s\right)-G\left(t_{1}, s\right)\right) f(s, x(s)) \mathrm{d} s\right| \leq \varepsilon M .
$$

Then, through the Arzela-Ascoli theorem, the operator $A$ is compact on $D$.

To summarize, $A: E \rightarrow E$ is completely continuous. The proof is complete.

Lemma 10. The operator $A$ is Frechét differentiable at $\infty$, and $A^{\prime}(\infty)=\beta T$. Proof. Since $A^{\prime}(\beta)=\lim _{x \rightarrow \infty} \sup _{t \in[0,1]} \frac{f(t, x)}{x}$, then for any $\varepsilon>0$ and $|x|>N$, there exists $N>0$ such that $\left|\frac{f(t, x)}{x}-\beta\right|<\frac{\varepsilon}{2}$, for any $t \in[0,1]$. Namely, $|f(t, x)-\beta x|<\frac{\varepsilon}{2}|x|$. Let $M_{0}=\max _{t \in[0,1],|x| \leq N}|f(t, x)-\beta x|$. Then for any $x \in \mathbb{R}$, $|f(t, x)-\beta x|<\frac{\varepsilon}{2}|x|+M_{0}$.

So we have

$$
|(A x-\beta T x)(t)|=\left|\int_{0}^{1} G(t, s)(f(s, x(s))-\beta x(s)) \mathrm{d} s\right| \leq\left(\frac{\varepsilon}{2}\|x\|+M_{0}\right) G_{0} .
$$

Thus, $\frac{\|(A x-\beta T x)\|}{\|x\|} \leq \frac{\left(\frac{\varepsilon}{2}\|x\|+M_{0}\right) G_{0}}{\|x\|}=\frac{\varepsilon}{2}+\frac{M_{0} G_{0}}{\|x\|}$.

For the above $\varepsilon$, there exists $N_{0}>0$ such that $\frac{M_{0} G_{0}}{\|x\|}<\frac{\varepsilon}{2}$, namely, $\frac{\|(A x-\beta T x)\|}{\|x\|} \leq \varepsilon$ for $\|x\|>N_{0}$. Thus, we can show

$$
\lim _{\|x\| \rightarrow \infty} \frac{\|A x-\beta T x\|}{\|x\|}=0
$$

The proof is complete.

Lemma 11. [13] Let $\Omega$ be a bounded open set in infinite dimensional real Banach space $E, \theta \notin \partial \Omega$ and $A: \bar{\Omega} \rightarrow E$ be completely continuous. Suppose that $\|A x\| \geq\|x\|, \quad A x \neq x, \forall x \in \partial \Omega$. Then $\operatorname{deg}(I-A, \Omega, \theta)=0$.

Lemma 12. [14] Let $A$ be a completely continuous operator which is defined on a Banach space $E$. Assume that 1 is not an eigenvalue of the asymptotic derivative. The completely continuous vector field $I-A$ is then nonsingular on spheres $S_{\rho}=\{x \mid\|x\|=\rho\}$ of sufficiently large radius $\rho$ and

$$
\operatorname{deg}(I-A, B(\theta, \rho), \theta)=(-1)^{k},
$$


where $k$ is the sum of the algebraic multiplicities of the real eigenvalues of $A^{\prime}(\infty)$ in $(1,+\infty)$.

Generalizing the previous lemmas, we obtain the following result.

Theorem 1. If (H1) and (H2) hold, then boundary value problem (1.1) has at least one nontrivial solution.

Proof. Obviously $B(\theta, r)$ is bounded open set, and $\theta \notin \partial(B(\theta, r))$. Via Lemma 9, we get $A: \overline{B(\theta, r)} \rightarrow E$ is completely continuous.

Combining (H2) and Lemma 10, we obtain the eigenvalue of $A^{\prime}(\infty)$ is $\frac{\beta}{\lambda} \neq 1$. Therefore, through Lemma 12, we get

$$
\operatorname{deg}(I-A, B(\theta, \rho), \theta)=(-1)^{k}
$$

By Lemma 7, we have $c=\sum_{n=0}^{\infty} \frac{\lambda^{n}}{n !} E_{2-\alpha, 1+\alpha n}^{(n)}(b)$. Therefore,

$$
\begin{aligned}
c & =\sum_{n=0}^{\infty} \frac{\lambda^{n}}{n !} E_{2-\alpha, 1+\alpha n}^{(n)}(b) \\
& =\sum_{n=0}^{\infty} \frac{\lambda^{n}}{n !} \sum_{j=0}^{\infty} \frac{b^{j} \Gamma(n+j+1)}{\Gamma(j+1) \Gamma((2-\alpha) j+2 n+1)} \\
& >\sum_{n=0}^{\infty} \frac{b^{n}}{\Gamma((2-\alpha) n+1)}=g_{1}^{\prime}(1) .
\end{aligned}
$$

Through the definition of $G(t, s)$ and Lemma 5, we get $G(t, s) \geq 0$ for $(t, s) \in[0,1] \times[0,1]$. Considering (H1), for any $\|x\|=r$,

$$
\begin{aligned}
|(A x)(1)| & =\int_{0}^{1} G(1, s) f(s, x(s)) \mathrm{d} s \\
& \geq \int_{0}^{\tau} g_{1}(1-s) f(s, x(s)) \mathrm{d} s \\
& \geq \tau g_{1}(1-\tau) \cdot \frac{r}{\tau g_{1}(1-\tau)}=\|x\| .
\end{aligned}
$$

i.e. $\|A x\| \geq\|x\|$. By Lemma 11, we get

$$
\operatorname{deg}(I-A, B(\theta, r), \theta)=0 .
$$

In conclusion, $\operatorname{deg}(I-A, B(\theta, \rho) \backslash B(\theta, r), \theta)=(-1)^{k}-0=(-1)^{k} \neq 0$.

So we get at least one $x \in B(\theta, \rho) \backslash B(\theta, r)$ is a fixed point of the operator $A$. That is to say, $x$ is one nontrivial solution of nonlinear problem (1.1). The proof is complete.

\section{Conclusion}

Theorem 1 is the main result of this paper. By Theorem 1, boundary value problem (1.1) has at least one nontrivial solution under the conditions of (H1) and $(\mathrm{H} 2)$. Because boundary value problem (1.1) has at least one nontrivial solution, we can investigate the state of the oscillator motion under this system in the later research. 


\section{Acknowledgements}

We thank the Editor and the referee for their comments.

\section{Conflicts of Interest}

The authors declare no conflicts of interest regarding the publication of this paper.

\section{References}

[1] Bai, Z., Chen, Y., Lian, H., et al. (2014) On the Existence of Blow up Solutions for a Class of Fractional Differential Equations. Fractional Calculus and Applied Analysis, 17, 1175-1187. https://doi.org/10.2478/s13540-014-0220-2

[2] Bai, Z., Dong, X. and Yin, C. (2016) Existence Results for Impulsive Nonlinear Fractional Differential Equation with Mixed Boundary Conditions. Boundary Value Problems, 2016, Article ID: 63. https://doi.org/10.1186/s13661-016-0573-z

[3] Liu, Y. (2016) New Existence Results for Positive Solutions of Boundary Value Problems for Coupled Systems of Multi-Term Fractional Differential Equations. Hacettepe Journal of Mathematics and Statistics, 45, 391-416. https://doi.org/10.15672/HJMS.20164512499

[4] Liu, X. and Jia, M. (2018) The Method of Lower and Upper Solutions for the General Boundary Value Problems of Fractional Differential Equations with P-Laplacian. Advances in Difference Equations, 2018, Article ID: 28. https://doi.org/10.1186/s13662-017-1446-1

[5] Han, Z., Lu, H. and Zhang, C. (2015) Positive Solutions for Eigenvalue Problems of Fractional Differential Equation with Generalized P-Laplacian. Applied Mathematics and Computation, 257, 526-536. https://doi.org/10.1016/j.amc.2015.01.013

[6] Liu, X., Jia, M. and Ge, W. (2017) The Method of Lower and Upper Solutions for Mixed Fractional Four-Point Boundary Value Problem with P-Laplacian Operator. Applied Mathematics Letters, 65, 56-62. https://doi.org/10.1016/j.aml.2016.10.001

[7] Jia, M. and Liu, X. (2014) Multiplicity of Solutions for Integral Boundary Value Problems of Fractional Differential Equations with Upper and Lower Solutions. Applied Mathematics and Computation, 232, 313-323. https://doi.org/10.1016/j.amc.2014.01.073

[8] Liu, X., Jia, M. and Ge, W. (2013) Multiple Solutions of a P-Laplacian Model Involving a Fractional Derivative. Advances in Difference Equations, 126, 1-12. https://doi.org/10.1186/1687-1847-2013-126

[9] Lutz, E. (2001) Fractional Langevin Equation. Physical Review E, 64, Article ID: 051106. https://doi.org/10.1103/PhysRevE.64.051106

[10] Podluny, I. (1999) Fractional Differential Equations. Academic Press, San Diego.

[11] Kilbas, A.A., Srivastava, H.M. and Trujillo, J.J. (2006) Theory and Applications of Fractional Differential Equations. Elsevier B V, Amsterdam.

[12] Diethelm, K. (2010) The Analysis of Fractional Differential Equations. Springer-Verlag, Berlin. https://doi.org/10.1007/978-3-642-14574-2

[13] Guo, D. and Lakshmikantham, V. (2014) Nonlinear Problems in Abstract Cones. Academic Press, Cambridge.

[14] Krasnosel'skii, M.A. and Zabreiko, P.P. (1984) Geometrical Methods of Nonlinear Analysis. Springer-Verlag, Berlin, Heidelberg. 